\title{
A procedure for studying strategy development in humans
}

\author{
FERNANDO A. GONZALEZ and MICHAEL CATHCART \\ CERT, Atlanta, Georgia, and Morris Brown College, Atlanta, Georgia
}

\begin{abstract}
An early version of the computer game Star Trek was modified to conduct behavioral research. By appropriate use of the starship's weapon and defense systems, the player can kill enemy ships and avoid destruction. The player's moves, as well as the time and state of the game environment when each move occurs, are recorded. The procedure tracks performance changes over time very well, and allows the dissection of performance into sequences of commands that reflect the subject's play strategy. Computer games such as Star Trek could be the basis for experiments that bridge the gap between operant and cognitive research.
\end{abstract}

The most challenging and interesting human tasks involve decision making based on incomplete information and inconstant contingencies. To perform such tasks well, the individual must learn to recognize stimuli that serve as cues for effective behaviors and, as conditions change, make the necessary adjustments to maintain a high likelihood of success. In this research, a modified version of the computer game Star Trek was used to study the development of play strategies and the sensitivity of the player to unannounced changes in contingencies. The game required the player to make decisions about engaging in any of several alternative behaviors that yielded outcomes that varied quantitatively or, sometimes, qualitatively, depending on circumstances beyond the player's control.

The research is in its early stages. A working version of the instrument (i.e., the game) has been completed, and some data have been obtained that indicate that the procedure tracks performance changes over time very well, and allows the dissection of performance into sequences of responses that reflect the subject's play strategy. These early results suggest that the game can be used to conduct experiments that bridge the gap between the operant and cognitive approaches to the study of behavior.

\section{METHOD}

\section{Software}

An early commercial version of a computer game based on the TV series "Star Trek" was modified for the present research. The original code, written for Apple computers in Apple Basic, was transcribed to Microsoft Quick Basic, and a shell was built around the game program to manipulate game parameters and record data. The essentials of the game have previously been described by Case,

The authors wish to thank David Case for providing the original version of the Star Trek game. This research was supported by Grant DAAL03-92-G-0382 from the U.S. Army Research Office to Morris Brown College. Reprints and copies of the program may be obtained from F. Gonzalez, Center of Excellence for Research on Training, Morris Brown College, 643 MLK Jr. Drive, NW, Atlanta, GA 30314 (e-mail: fgcert@aol.com).
Ploog, and Fantino (1990; see also Case, 1995). The game was simplified by eliminating several superfluous commands and programmed outcomes.

In this game, the player is in charge of the starship Enterprise. Her or his mission is to seek and destroy Klingon warships. The Enterprise can move across sectors and quadrants of the universe searching for Klingon vessels. A short-range scanner shows the objects (Klingons, stars, and bases) in the quadrant occupied by the Enterprise. Other commands provide a more extensive, but less detailed, view of the universe. The Enterprise leaves its base with an energy charge that can only be replaced by docking on another Federation base. All commands use up energy.

The Enterprise has two weapons systems: phasers and torpedoes. Phasers propagate in all directions and, if sufficiently strong, destroy all Klingons in the local quadrant. Torpedoes must be aimed in the direction of the vessel to be destroyed. In this game, when Klingon numbers decrease sufficiently, they are replaced by new vessels that appear unpredictably outside the range of the Enterprise's long-range scanners. Thus, the density of targets remains approximately constant throughout the game.

The Enterprise's force shield partially defends it from Klingon attacks. The shield must be lowered to use phasers, but torpedoes can be fired with the shield raised. Klingon blasts can severely harm the $E n$ terprise if the shield is down. The effects of Klingon attacks are always spatially and temporally cumulative. The Enterprise is lost if it uses up all its energy or if it is sufficiently damaged by the Klingons.

Events in this game occur serially rather than continuously. For example, if the player enters a command to move to a new quadrant, the move is immediately carried out and the player is informed about the consequences of the move, including the effects of Klingon attacks on the Enterprise. Nothing then happens until the next command is issued. Therefore, since game duration is fixed, the longer the time taken to make a command decision and to execute it, the fewer opportunities there are for killing Klingons. Feedback is constantly provided concerning the strength of nearby Klingons, the number of kills, the available energy, damage to the ship's systems, and the number of unused torpedoes. All moves, defenses, attacks, scans, repairs, and so forth require a command and the specification of relevant details, such as the amount of energy to be used.

\section{Subjects and Procedure}

Three college students were paid \$5.75 per hour to play the game, and were hired to play $10-12 \mathrm{~h}$ per week. They played in a small room containing the computer and a comfortable chair. The players were provided with a detailed set of typed instructions describing the game's environment and the operation of the commands, 
and explaining the goals of the game. The instructions did not provide information about strategy; the players had to figure out on their own how to accomplish the game's mission. Games lasted 20 min or until the Enterprise was destroyed.

All play commands were entered from the keyboard, and involved depressing two letter keys and no more than four number keys to indicate energy, coordinates, and so forth. A student assistant supervised the initiation of each experimental session. Several games were usually played per session, and all games were played to completion. The subjects were briefly interviewed every 30 or so games to assess their understanding of the game and their willingness to continue participating in the experiment. The questions were always the same and were very general, such as, "Have you settled on a way to play the game?", "Have you changed your way of playing since last time we talked?", and "How do you feel about playing twenty more games?"

Ten different commands could be entered, and there were no sequencing restrictions. Half of the commands could impact the game environment. When the player entered a command, the command type, the time, the position of the Enterprise, the amount of energy available, the number of torpedoes available, the number of Klingons in mission area, the number of killed Klingons, and the number of accessible bases were recorded in a sequential data file. A data-analysis program generated 63 descriptive statistics summarizing each session's data. The same program yielded the frequency distribution of command sequences and the frequency of kills associated with each sequence. Also generated were the frequency distribution of command sequences of different lengths and the associated frequency of kills.

\section{RESULTS}

The three subjects played 64,124 , and 320 games, respectively. The present paper will emphasize the data from only one subject, M.A.C. Figure 1 shows that M.A.C.'s kill and survival statistics seemed to continue to improve even after 300 sessions. However, the increases in kill rate after the first 50-75 sessions reflected not improved play strategy but, rather, an increase in the rate of command execution (see Figure 2). That is, M.A.C. got better at killing Klingons because, with practice, he executed commands more quickly; he did not necessarily use more effective command sequences.

Figure 3 indicates that for M.A.C., increases in torpedo (PT) kill rates (lower panel) were mostly due to increases in the rate of execution of PT commands (upper panel). On the other hand, his proficiency at using the phaser (PH) increased as the experiment progressed (middle panel), but, surprisingly, his rate of execution of this command decreased.

Play strategies were denoted by the frequency distributions of command sequences. For the purpose of analysis, command sequences started with a short-range scan (SR) and ended with the command that preceded the next SR. The SR command provided a static view of the local quadrant and was a necessary precursor to the effective execution of most other commands; for example, torpedoes could not be aimed unless the player first executed the SR command to obtain a view of the position of the Klingons in relation to the Enterprise. All subjects used this command about twice as often as any other command.

The distributions of command sequences from the early sessions were erratic, but became more regular across
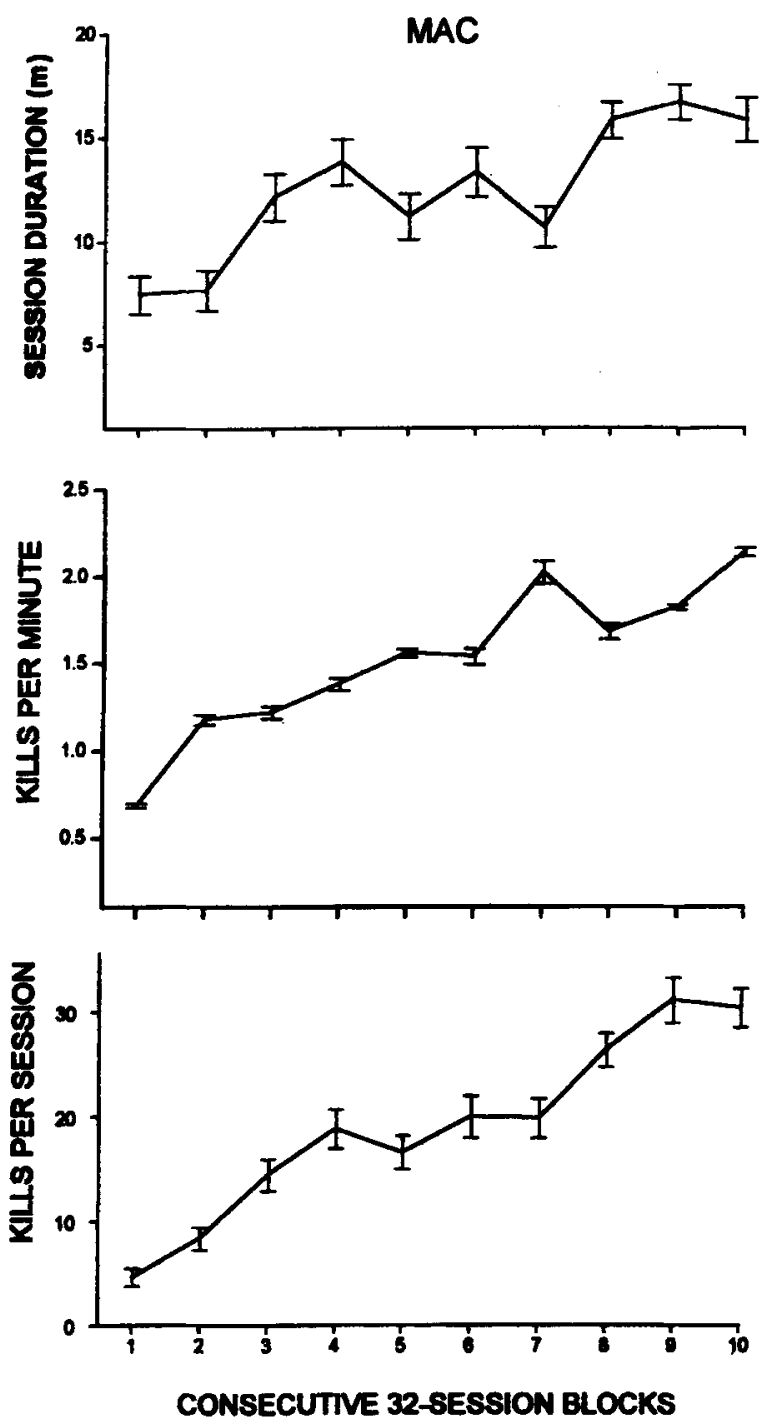

Figure 1. Means $\pm S D$ ( $y$-axes) of game duration (upper panel), kill rate (middle panel), and kills per session (lower panel) in each of 10 blocks of 32 sessions ( $x$-axes) for Subject M.A.C. The 320 sessions represent about $80 \mathrm{~h}$ of game playing. Performance improved slowly throughout the experiment.

games as the subjects gained experience, with the same five or six sequences occurring most frequently. The distributions stabilized after about 50-75 games, denoting fairly fixed patterns of command choices following each SR. Figure 4 shows the relative frequency of occurrence of the 13 command sequences used most frequently by M.A.C. during the last 23 sessions, and the relative frequency of the same sequences during 23 sessions at the beginning and in the middle of the experiment. These same 13 sequences accounted for about $55 \%$ of all of M.A.C.'s sequences throughout the experiment.

Thus far, only M.A.C. has been exposed to a change in contingencies. From Sessions 174-204, without informing him, PH effectiveness was increased by $50 \%$. It was anticipated that this would substantially increase his 


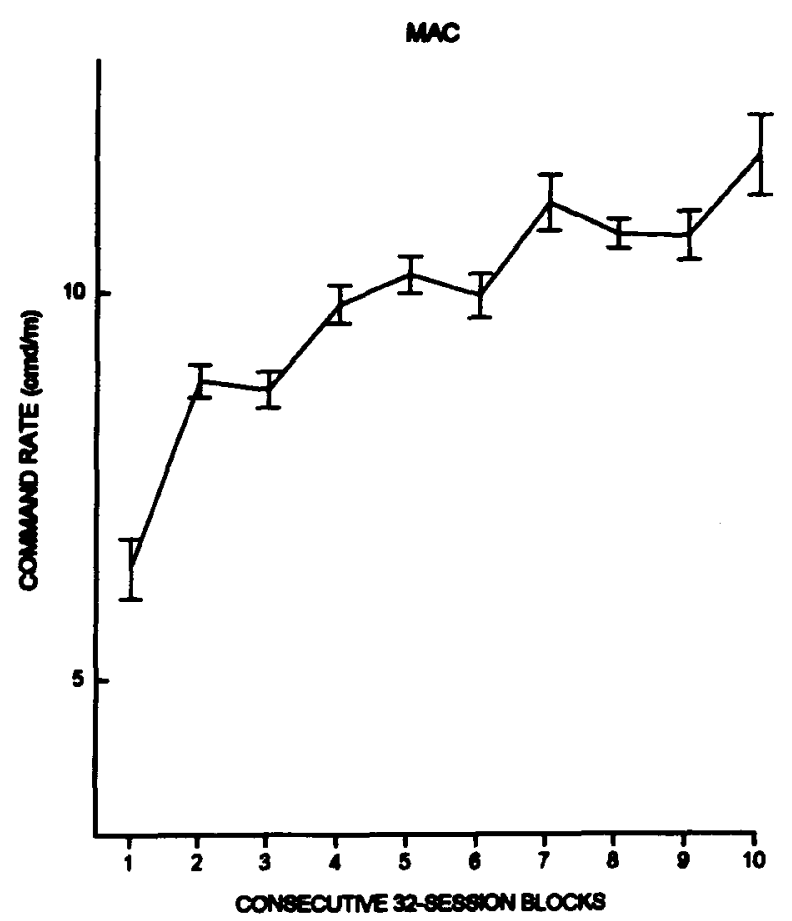

Figure 2. Mean $\pm S D$ of the rate of execution of all commands $(y$-axis) in each of 10 blocks of 32 sessions. Most commands were executed faster as the experiment progressed.

rate of PH. Figure 3 shows that there was indeed an increase in the rates of $\mathrm{PH}$ use and $\mathrm{PH}$ kills, leading, as denoted in the middle panel of Figure 1, to an increase in total kill rate. However, these increases were associated with decreases in game duration (Figure 1, top panel) and, as a result, the frequency of kills per session did not increase (Figure 1, lower panel). M.A.C.'s play returned to premanipulation form soon after the effectiveness of $\mathrm{PH}$ was returned to the original level. Interviews during and after the change in PH effectiveness suggested that M.A.C. was unaware of the change. In a more detailed final interview, M.A.C. confirmed that he had failed to notice a change in the game, and stated that he did not alter his way of playing during those sessions.

The ratio of torpedo commands to phaser commands (PT/PH) during M.A.C.'s last 10 sessions was about 7:1. This was consistent with his concern, expressed during the final interview, that lowering the shields to use phasers entailed "too high a risk of destruction" of the Enterprise. Hence, he evidently adopted a play strategy that emphasized the ship's safety but that resulted in kill rates that were approximately $20 \%$ lower than those of Subject M.D.P., whose PT/PH ratio was 4:1 during his final 10 sessions (Sessions 115-124), and who used phasers "whenever the energy reserves were high and there were few Klingons around." Figure 5 shows that M.A.C. and M.D.P. evinced different distributions of the more frequently used sequences, reflecting differences in their play strategies. This is in clear contrast to the similarities among the distribution of M.A.C.'s command sequences at different stages of the experiment (Figure 4), which denote M.A.C.'s consistently conservative play strategy.

\section{DISCUSSION}

The purpose of this paper is to show how a computer game can be effectively used to generate intricate behavioral data that can be analyzed to investigate phenomena of interest to researchers with different theoretical bi-
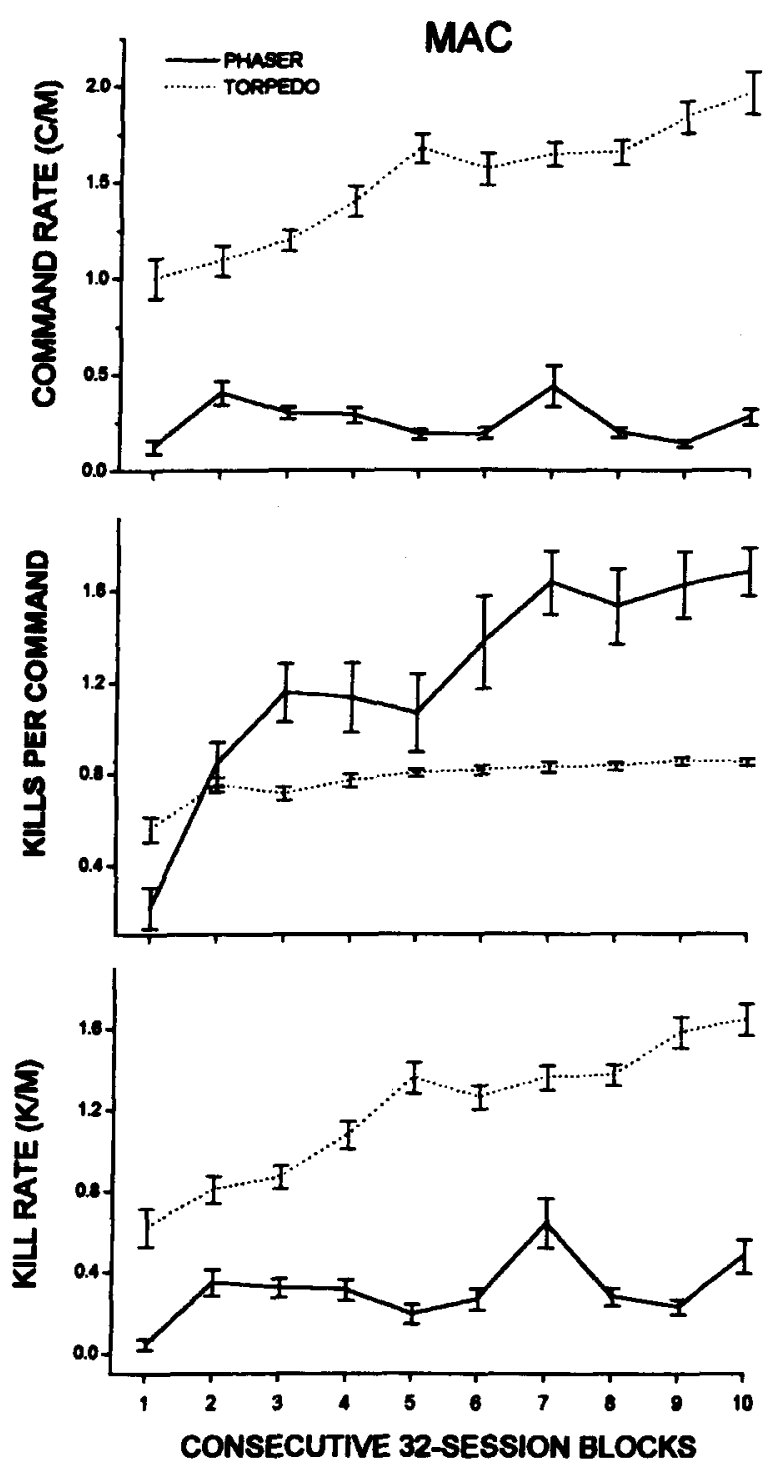

Figure 3. Means $\pm S D$ ( $y$-axes) of rate of execution of phaser command and of torpedo command (upper panel), ratio of kills per phaser command and per torpedo command (middle panel), and rate of kills attained with the phaser command and the torpedo command (lower panel) in each of 10 blocks of 32 sessions ( $x$-axes) for Subject M.A.C. Increases in torpedo kill rates were due to increases in torpedo command rates. Phaser kill rates did not increase substantially because increases in phaser kill effectiveness (kills per phaser command) were accompanied by decreases in phaser command rate. 


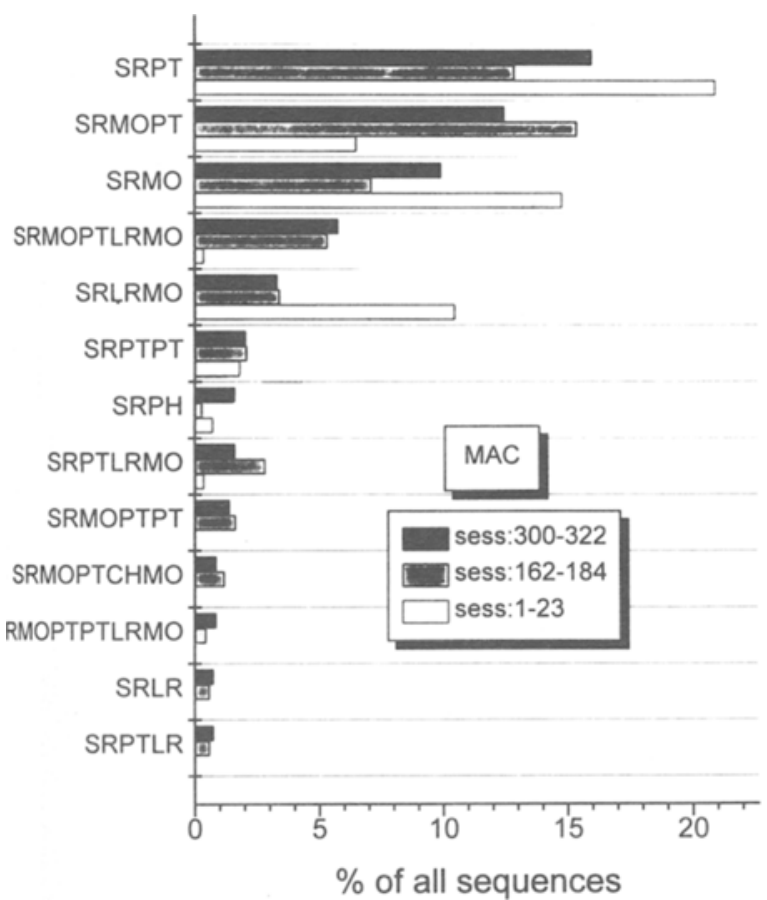

Figure 4. Percentage distribution of the 13 most frequent sequences of commands executed by Subject M.A.C. during blocks of 23 sessions at the beginning, middle, and end of the experiment. Commands: SR, short-range scan; PT, torpedo; MO, move; LR, long-range scan; PH, phaser; CH, star chart. The same 13 sequences accounted for about $55 \%$ of all sequences throughout the experiment.

ases. Thus, in this study, the data are amenable to an analysis of rate of responding or choice of behavior alternatives that is typical of the operant perspective, but the same data are also amenable to a stochastic process analysis of behavior sequences to uncover global play strategies, which is more typical of the cognitive psychology perspective.

The method used in this study is typical of operantconditioning research, in that (1) few subjects were studied over many sessions; (2) minimal initial instructions described only the mechanical aspects of the procedure that allowed the subjects to begin to play the game; and (3) the dependent variables were measures of gamerelevant behaviors that were recorded automatically and continuously by the apparatus. The topic of the study human judgment and strategy formation-on the other hand, is more typical of cognitive psychology than of operant-conditioning research. The vast amounts of behavioral data generated with the modified Star Trek game can be analyzed by operant psychologists to expose response patterns and the control exerted by events in the programmed environment, or they can be analyzed by cognitive psychologists to uncover the underlying cognitive processes that govern actions.

Other participants in this symposium have discussed the use and advantages of computer games for conducting psychological research in training (Donchin, 1995), for problem solving (Porter, 1995), for operant condition- ing (Case, 1995), and for comparative cognitive processes (Washburn \& Gulledge, 1995). From the topics addressed, it is apparent that computer games allow investigators to conduct a range of studies on factors affecting the acquisition, asymptotic characteristics, and maintenance of complex human (and monkey) behaviors or cognitive skills in a very objective fashion. The point is that computer games can be used to conduct research in a variety of theoretical contexts, and that because the tasks and recorded behaviors can be very complex, the same tool can be used to conduct studies pertinent to different theoretical perspectives and may serve to clarify the differences and similarities among them.

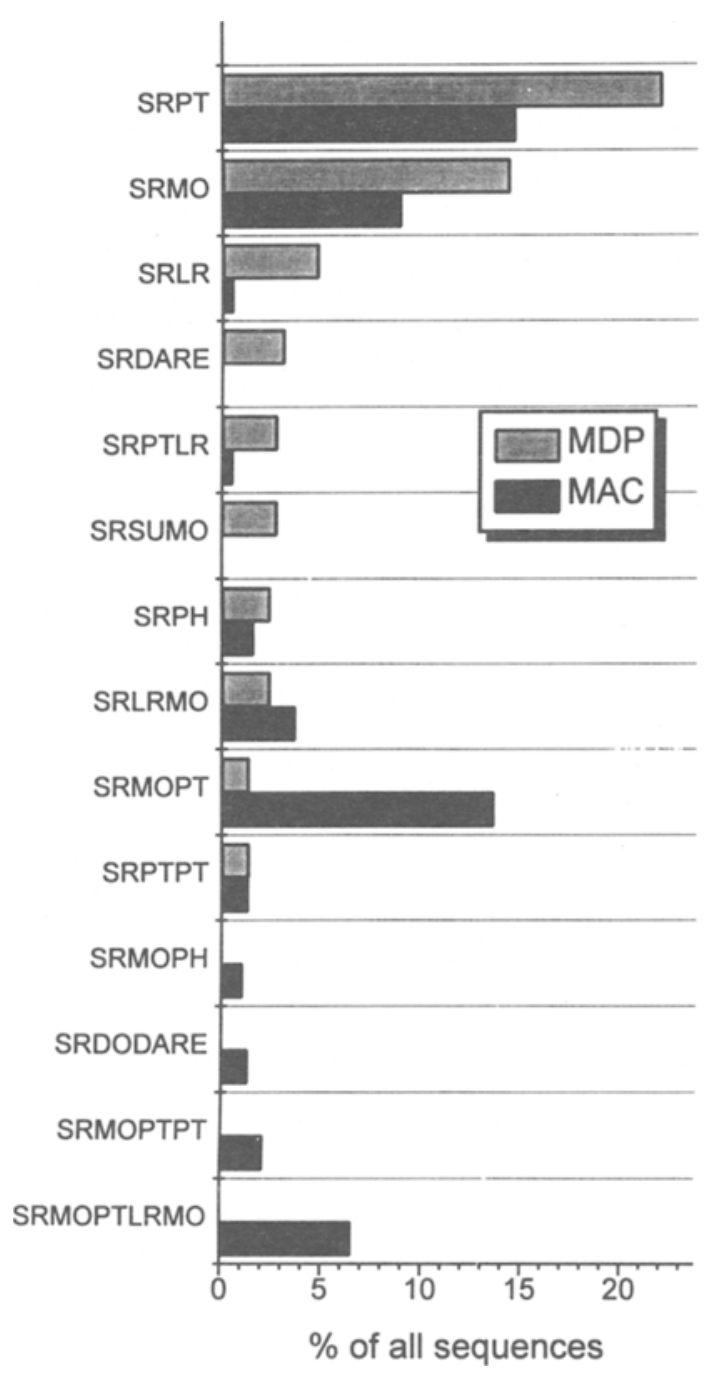

Figure 5. Percentage distributions of the 10 sequences used most frequently by Subjects M.D.P. and M.A.C. during the last 10 sessions of the experiment. Sequences are arranged in descending order of frequency for Subject M.D.P. Commands: DA, damage report, RE, repair, SU, shield up, DO, dock; others are as in Figure 4. Subject M.D.P. averaged $20 \%$ more kills than Subject M.A.C. His sequences were generally shorter, he used the $L R$ and $P H$ commands more frequently, and he moved more often than Subject M.A.C. across quadrants in an effort to avoid the more dangerous encounters. 


\section{REFERENCES}

CASE, D. A. (1995). On trekking to operant frontiers. Behavior Research Methods, Instruments, \& Computers, 27, 211-216.

CASE, D. A., Ploog, B. O., \& Fantino, E. (1990). Observing behaviot in a computer game. Journal of the Experimental Analysis of Behavior, 54, 185-199.

Donchin, E. (1995). Video games as research tools: The Space Fortress game. Behavior Research Methods, Instruments, \& Computers, 27, 217-223.
PORTER, D. B. (1995). Computer games: Paradigms of opportunity. Behavior Research Methods, Instruments, \& Computers, 27, 229-234.

WASHBURN, D. A., \& GULLEDGE, J. P. (1995). Game-like tasks for comparative research: Leveling the playing field. Behavior Research Methods, Instruments, \& Computers, 27, 235-238.

(Manuscript received November 21, 1994; revision accepted for publication February 17, 1995.) 